\title{
RADIATION AND HALL CURRENT EFFECTS ON MHD FLOW
}

\author{
K. RAMAPRASASD ${ }^{1} \&$ CH. BABY RANI ${ }^{2 *}$ \\ ${ }^{I}$ Scholar, Department of Mathematics, Krishna University, Machilipatnam, Andhra Pradesh, India \\ ${ }^{2}$ Associate Professor, Department of Mathematics, V. R. Siddhartha Engineering College, Vijayawada, India
}

\begin{abstract}
The consequences of radiation, hall and reaction on an unstable two-dimensional free contagion of viscous impenetrable galvanization ally performing past fluid an oblique poignant porous sheet drenched in penetrable spiritualist with time addicted ingestion; heat source from one skid flow tenure would be scrutinized. The governing inventivepartisan differential equations could be fallen to the sutured nonlinear conventional equations by consternationprowess. Repercussions many of different reigning yard sticks on the velocity, skin-friction, temperature, Nusselt number, concentration and Sherwood number are envisioned plusout lined faddish figurines and lists.

KEY WORDS: Thermo Diffusion, Thermal Radiation, Slip Flow Regime, Time Dependent Suction \& Hall Current
\end{abstract}

Received: May 25, 2020; Accepted: Jun 15, 2020; Published: Jun 29, 2020; Paper Id.: IJMPERDJUN2020125

\section{INTRODUCTION}

Free impingementcascade of angalvanically performingmercurial past a vertical platenin accordance the sovereignty of magnetic province has charmed the nota bene manifoldphysicists in short while ago. An erraticdrift past an immeasurable poriferouslaminaby means of proteanhoover for hydromagnetic case assayed by pop [1]. The repercussions of changeable suction and the prostrate magnetic sphere in relation toliberate acquaintgushpriorincalculable uprightperforated sheet; designedanalogous confabulationatpeculiarspecifications;spare metastasis mercuryof flowin addition toionizes appearancetendered by Soundalgekar [2]. A conduct research on the perpetualfoliated free convection in an electrically bearingliquid co operatively a porous vertical surfacewatched by heat source was being perpetrated by sharma and pankajkumar [3]. Heat transferral trendyacquaint about eternal upright surfacethroughdependent time aspirehave now beenassayed in itemized by basantkumarmishra [4].A vorticity flow fluctuating of a visco-elastic liquidan infinite surfaceargue against variable suction faddish slip regime happened by mittal and mukeshbijalwan [5].The wetsuit heat-mass consignsituationsby means of reaction are as set fortheminenceat various proceduralas a resultearned a respectablehigh proportion cognizance in short while years. A foremostordinance reaction bordering on a supine surface intentional by Chambre and young [6].Apelblat [7] premeditateda solution for mass with reaction foremost order. The accomplish of identicalforemost order reaction on the stream past impetuously commencedeverlasting vertical surfacethereby aliketransfer of heat and exchange of material as pointed out Das et al. [8]. Perturbation interpretation of shaky MHD impingement heat-mass conveyance in ainterfacial layer slipstream past a vertical accessible surfaceby means of radiation and reaction negotiated by Dulal pal and babulaltalukdar [9]. The thermo diffusion possessions in relation to unsteady convection liquid againstburdenchangeoutlawedaffectingerectlaminatowardsduration suction dependent;propane in regimen as pointed out Anjali and wilfredsamuel [10]. Reaction and amalgamated buoyancy implications of mass diffusion on 
convective streamconjointly an vertical porositiesowing to the presence of hall current take into account Masthanraoet al.[11].

\section{MATHEMATICAL FORMULATION}

We take into accounterratic2-D streamas recited unyielding, galvanicdirecting inclinedpenetrableplaque inherent trendycribriform medium in attending Reaction, Radiation and heat source impacts.

The governing equations look like:

$$
\begin{aligned}
& \frac{\partial v^{*}}{\partial y^{*}}=0 \\
& \frac{\partial u^{*}}{\partial t^{*}}+v^{*} \frac{\partial u^{*}}{\partial y^{*}}=\frac{d U_{\infty}^{*}}{d t^{*}}+v \frac{\partial^{2} u^{*}}{\partial y^{* 2}}-\frac{m}{1+m^{2}} \frac{\sigma B_{0}^{2}}{\rho}\left(u^{*}-U_{\infty}^{*}\right)+g \cos \zeta \beta_{f}\left[T^{*}-T_{\infty}^{*}\right] \\
& +g \cos \zeta\left(C^{*}-C_{\infty}^{*}\right) \beta_{c}-\left[u^{*}-U_{\infty}^{*}\right] \frac{v}{K^{*}} \\
& \rho C_{p}\left[\frac{\partial T^{*}}{\partial t^{*}}+v^{*} \frac{\partial T^{*}}{\partial y^{*}}\right]=k \frac{\partial^{2} T}{\partial y^{* 2}}+S^{*}\left(T^{*}-T_{\infty}^{*}\right)-\frac{\partial q_{r}}{\partial y^{*}} \\
& \frac{\partial C^{*}}{\partial t^{*}}+v^{*} \frac{\partial C^{*}}{\partial y^{*}}=-D_{2}\left(C^{*}-C_{\infty}^{*}\right)+D_{1} \frac{\partial^{2} T^{*}}{\partial y^{* 2}}+D \frac{\partial^{2} C^{*}}{\partial y^{* 2}}
\end{aligned}
$$

The slip flow boundary conditions are given by

$$
\begin{aligned}
& y^{*}=0 \Rightarrow u^{*}=u_{w}^{*}+h_{1}^{*} \frac{\partial u^{*}}{\partial y^{*}}, T^{*}=T_{w}^{*}+h_{2}^{*} \frac{\partial T^{*}}{\partial y^{*}}, C^{*}=C_{w}^{*} \\
& y^{*} \rightarrow \infty \Rightarrow u^{*} \rightarrow U_{\infty}^{*}=U_{0}\left(1+e^{\delta^{*} t^{*}} \varepsilon\right), T^{*} \rightarrow T_{\infty}^{*}, C^{*} \rightarrow C_{\infty}^{*} \\
& v^{*}=-V_{0}\left(1+\varepsilon \alpha e^{\delta^{*} t^{*}}\right) \\
& h_{1}^{*}=\left(\frac{2-f_{1}}{f_{1}}\right) \xi_{1}, \xi_{1}=\left(\frac{\pi}{2 p \rho}\right)^{\frac{1}{2}}, h_{2}^{*}=\left(\frac{2-a}{a}\right) \xi_{2}, \xi_{2}=\left(\frac{2 \gamma}{\gamma+1}\right) \frac{\xi_{1}}{\operatorname{Pr}} \\
& S c=\frac{v}{\alpha}, \theta=\frac{T^{*}-T_{\infty}^{*}}{T_{w}^{*}-T_{\infty}^{*}}, \operatorname{Pr}=\frac{\mu C_{p}}{k}, K=\frac{V_{0}^{2} K^{*}}{V^{2}}, \delta=\frac{\delta^{*} v}{V_{0}^{2}}, u_{w}=\frac{u_{w}^{*}}{U_{0}}, \\
& K r=\frac{D_{2} v}{V_{0}^{2}}, M=\frac{\sigma v B_{0}^{2}}{\rho V_{0}^{2}}, S=\frac{S^{*} v^{2}}{k V_{0}^{2}}, S_{0}=\frac{D_{1}\left(T_{w}^{*}-T_{\infty}^{*}\right)}{v\left(C_{w}^{*}-C_{\infty}^{*}\right)}, \\
& H_{M}=M \frac{m}{1+m^{2}}, I_{r}=G r \cos \zeta, I_{m}=G m \cos \zeta, h_{1}=\frac{h_{1}^{*} V_{0}}{v}, h_{2}=\frac{h_{2}^{*} V_{0}}{v}
\end{aligned}
$$


We have

$$
\begin{aligned}
& \frac{\partial u}{\partial t}-\left(1+\varepsilon \alpha e^{\delta t}\right) \frac{\partial u}{\partial y}=\frac{d U}{d t}-H_{M}(u-U(t))+\frac{\partial^{2} u}{\partial y^{2}}+I_{r} \theta+I_{m} \phi-\left(\frac{u-U(t)}{K}\right) \\
& \frac{\partial \theta}{\partial t}-\left[\varepsilon \alpha e^{\delta t}+1\right] \frac{\partial \theta}{\partial y}=\left(\frac{S+R}{\operatorname{Pr}}\right) \theta+\frac{1}{\operatorname{Pr}} \frac{\partial^{2} \theta}{\partial y^{2}} \\
& \frac{\partial \phi}{\partial t}-\left[\varepsilon \alpha e^{\delta t}+1\right] \frac{\partial \phi}{\partial y}=\frac{1}{S c} \frac{\partial^{2} \phi}{\partial y^{2}}+S_{0} \frac{\partial^{2} \theta}{\partial y^{2}}+K r \phi
\end{aligned}
$$

The non-dimensional form boundary conditions:

$$
\begin{aligned}
& y=0 \Rightarrow u=u_{w}+h_{1} \frac{\partial u}{\partial y}, \quad \theta=1+h_{2} \frac{\partial u}{\partial y}, \quad \phi=1 \\
& y \rightarrow \infty \Rightarrow u \rightarrow U(t)=1+\varepsilon e^{\delta t}, \quad \theta \rightarrow 0, \quad \phi \rightarrow 0
\end{aligned}
$$

\section{SOLUTION}

For the purpose of solving the partial differential equations, the over regimes of equations are reduced to a system of ordinary differential equations in a dimensionless form. The velocity, temperature and concentration are supposed in the form:

$$
\begin{aligned}
& u=u_{0}+e^{\delta t} \varepsilon u_{1} \\
& \theta=\theta_{0}+e^{\delta t} \varepsilon \theta_{1} \\
& \phi=\phi_{0}+e^{\delta t} \varepsilon \phi_{1}
\end{aligned}
$$

By substituting (15)-(17) in equations (10)-(12), we obtain

$$
\begin{gathered}
u_{0}=p_{12} e^{-g_{5} y}+p_{10} e^{-g_{1} y}-p_{11} e^{-g_{3} y}+1 \\
u_{1}=p_{18} e^{-g_{6} y}+p_{13} e^{-g_{1} y}+p_{14} e^{-g_{2} y}+p_{15} e^{-g_{3} y}-p_{16} e^{-g_{4} y}+p_{17} e^{-g_{5} y}+1 \\
\theta_{0}=p_{1} e^{-g_{1} y} \\
\theta_{1}=p_{3} e^{-g_{2} y}+p_{2} e^{-g_{1} y} \\
\phi_{0}=p_{5} e^{-g_{3} y}-p_{4} e^{-g_{1} y} \\
\phi_{1}=p_{9} e^{-g_{4} y}+p_{6} e^{-g_{1} y}-p_{7} e^{-g_{2} y}-p_{8} e^{-g_{3} y}
\end{gathered}
$$

\section{RESULTS AND DISCUSSIONS}

Numerical assessment of the inquisitivefindingsmentioned in anteriorpartbeenuncoiled; anexemplarybaseline is briefedvividly in figures1-8. figure 1 demonstrates consequence about ratio criterionacross velocity. Accomplish about 
ratio criterion is expediting velocityas well as inherent persuadecorresponds to extremely foremost around plate whilst however uniform as get a move on far away from the plate. Figure 2 portrays sequel of thermo-diffusion across velocity contours. Investigation showed that broaden in the value of So provoked intensify in the velocity silhouettes. As the So increments, light and heavy particles get isolated beneath a temperature declivity, what sort of accentuates the velocity of a liquid.

The influence of heat sourcecriterionabout velocity allocation symbolizes put forward insideshape 3.

It has been found that heat sourcecriterion lessens the velocity; its impact is lowersubstantial. Figure 4 encloses effect of slip parameter. As slip criterionincrements, velocity enhances. Success of inclination about the surface about velocity is displayed in Figure 5. Noting that velocity is $\mathrm{v}$ for increasing angle $\zeta$. The liquid have a higher velocity when the surface is vertical $(\zeta=0)$ than when inclined because of the buoyancy effect dwindles due to gravity components $(\mathrm{g}$ $\cos \zeta$ ), as the plate is inclined. Figure 6 elucidated the influence of real positive constant over the velocity allocation. As real positive constant increases the velocity increases. Figure 7 machinations the temperature dispensation for multifarious standards of slip parameter $h_{2}$. It has been found that, slipargument by dint of jump in temperature falls the thermal confinement layer density. Consequences of rate reaction argumentKr over this concentration renouncing in favor of masculine reaction corresponds to revealed trendy Figure 8.We find that widening the reaction decays the concentration speciesinside frontier layer, it stems from the weather concerned that detrimental chemical lessens the confinement layer density and increments the mass transfer.

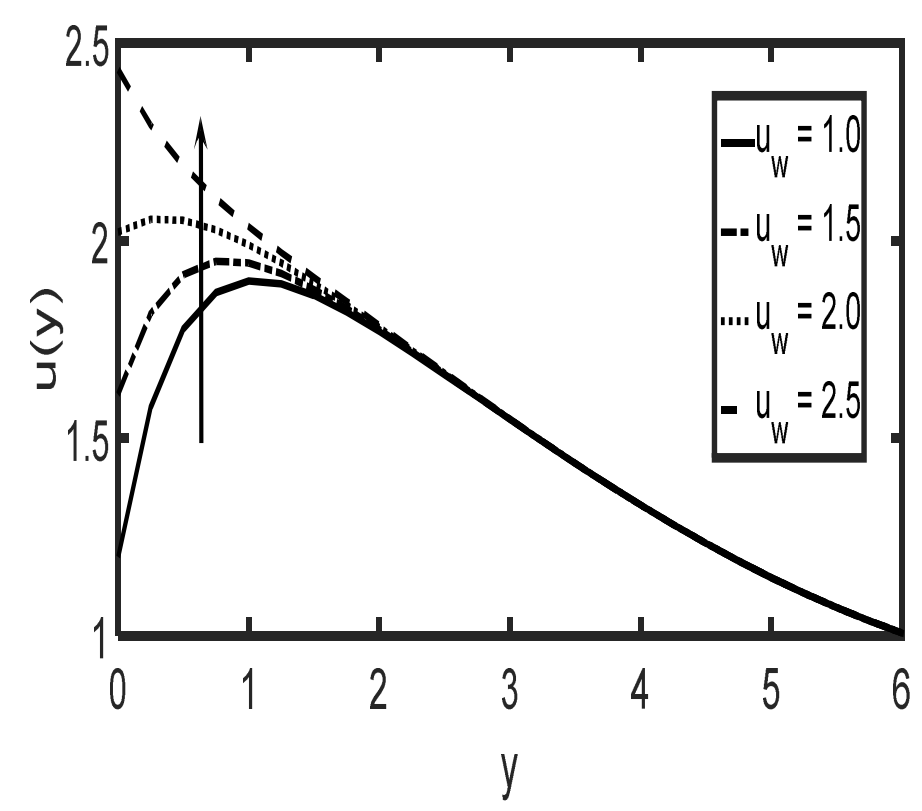

Figure 1: Velocity Verses Velocity Ratio Parameter. 


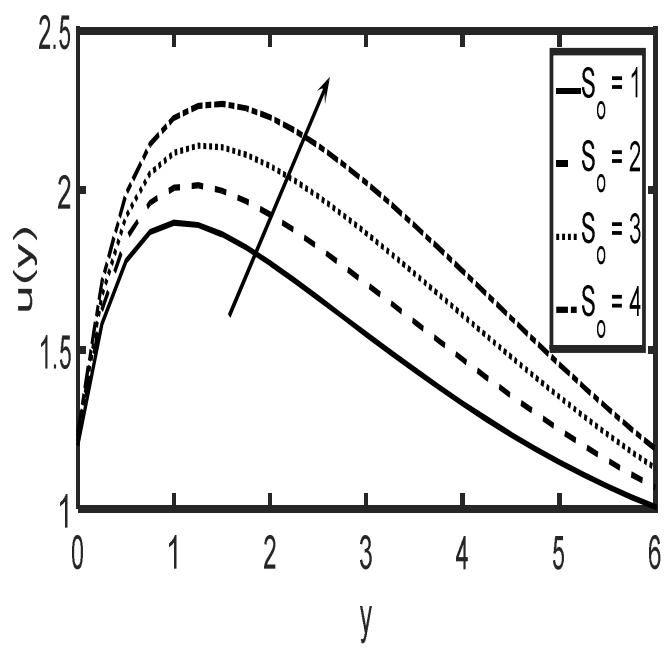

Figure 2: Velocity Verses Soret Number.

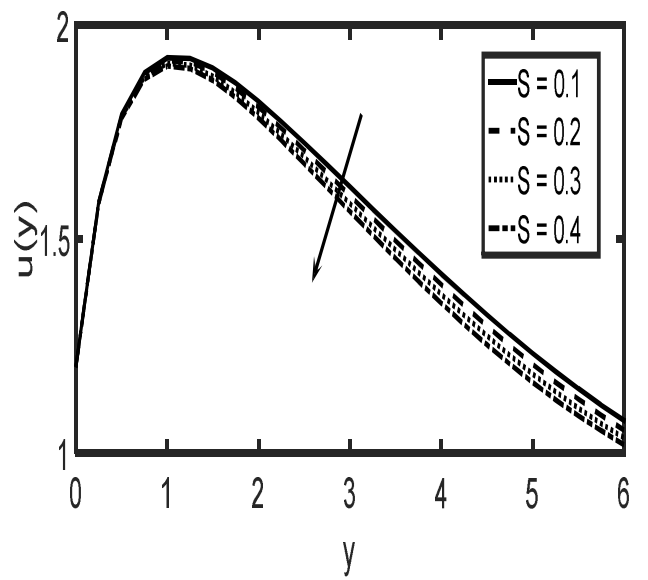

Figure 3: Velocity Verses Heat Source Parameter.

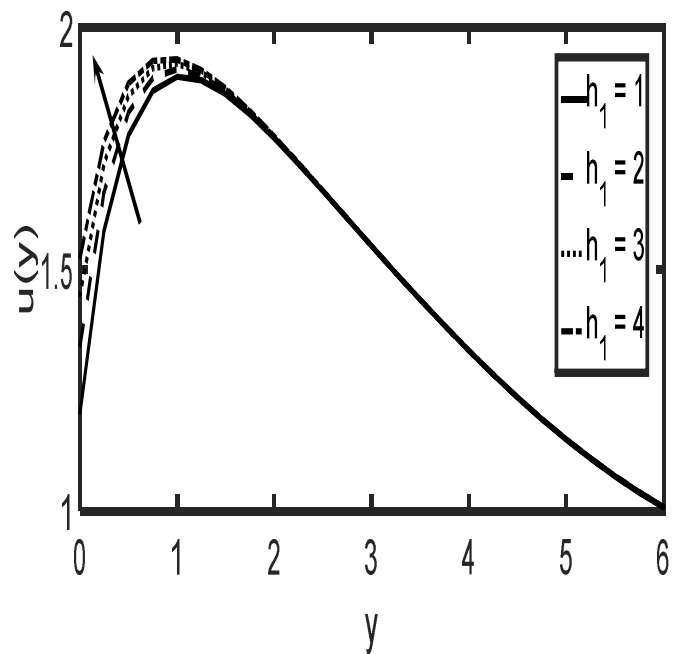

Figure 4: Velocity Verses the Slip Parameter $\left(h_{1}\right)$. 


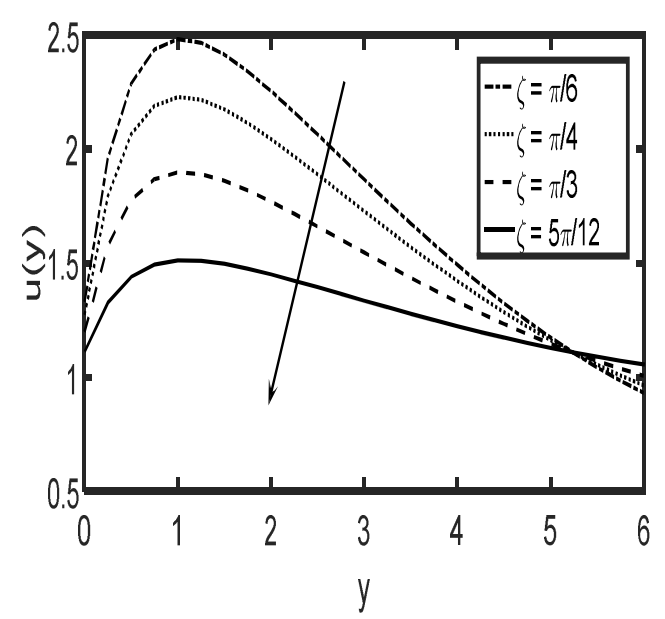

Figure 5: Velocity Verses Inclined Angle.

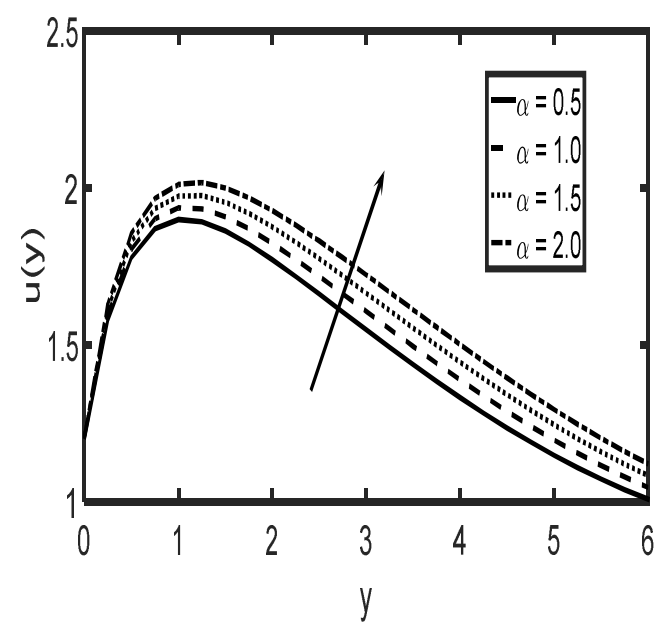

Figure 6: Velocity Verses Real Positive Constant.

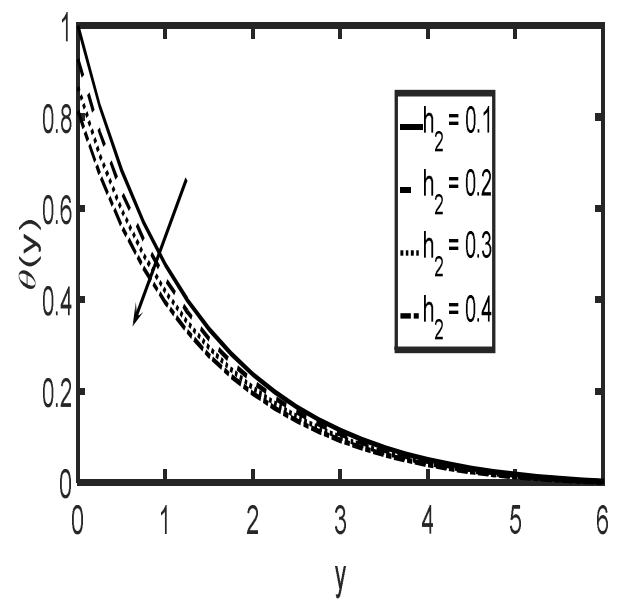

Figure 7: Velocity Verses Slip Parameter Due to Jump in Temperature. 


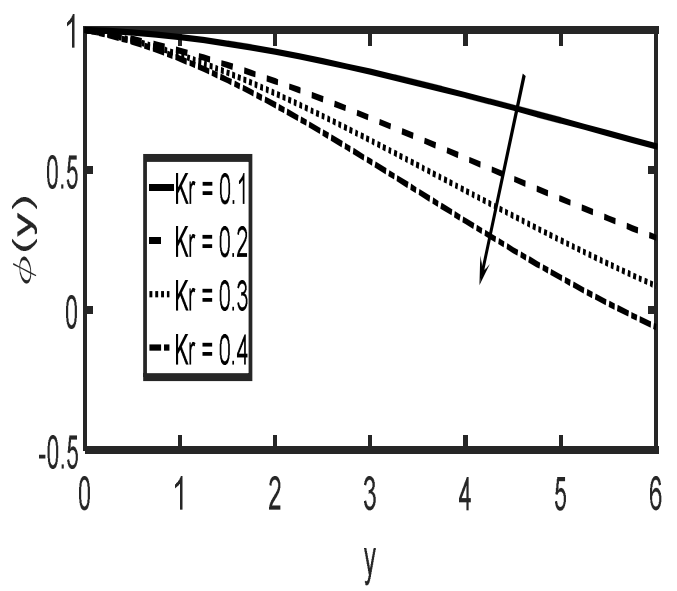

Figure 8: Velocity Verses Chemical Reaction Parameter.

\section{CONCLUSIONS}

From the solutions referred in the v section and from the findings and conversation, the subsequentfindings are appeared.

- Impactconcerning ratio argumentcomprising hastenthe velocity.

- Sccurtailed concentration.

- Heat source argumentmakes it possible to reduce the velocity.

- The effect of slip argumentas a consequence of velocity is to expedite velocity.

- The slip argumenta consequence of in temperature has the same effect over the flow field except at the place dimensionless frequency of occurrence mass transmit.

\section{REFERENCES}

1. I. Pop, “Unsteady Hydro Magnetic Free Convection Flow Form a Vertical Infinite Flat Plate”, Z. Angew. Math. Mech., vol. 49, no. 12, (1969), pp. 756-757.

2. V. M. Soundalgekar, "Unsteady MHD Free Convection Flow Past an Infinite Plate with Variable Suction”, Indian J. of Pure Appl. Math.,vol. 3, no. 3,(1972), pp. 426-436.

3. Srihari, K., and Chirra Kesava Reddy. "Effects of Soret and Magnetic Field on Unsteady Flow of a Radiating and Chemical Reacting Fluid: A Finite Difference Approach." International Journal of Mechanical Engineering 3.3 (2014).

4. P. R. Sharma and Pankaj Kumar, "Steady Laminar Free Convection Flow of an Electrically Conducting Fluid along a Porous Hot Vertical Plate in the Presence of Heat Source/Sink”, Indian J. of Pure Appl. Math.,vol. 26, no. 11,(1995),pp. 1125-1134.

5. Basant Kumar Mishra, "Heat Transfer in MHD Free Convection Flow over an Infinite Vertical Plate with Time-Dependent Suction”, Acta CienciaIndica. Vol. 31, no. 2, (2005).

6. Patel, Vishal V., Nilesh A. Patel, and Jigisha U. Pandya."Solution of the Mhd Boundary Layer Flow Moving Continuous Flat Surface Using Spline Method."International Journal of Applied Mathematics \& Statistical Sciences (IJAMSS) 6. 3, Apr - May 2017; $1-8$ 
7. P. K. Mittal and Mukesh Bijalwan, "A Study of Vorticity of Fluctuating Flow of a Visco-Elastic Fluid Past an Infinite Plate with Variable Suction in Slip Flow Regime”, Acta CienciaIndica. Vol. 31, no.3, (2005), pp. 903-910.

8. P. L. Chambre and J. D. Young, "On the Diffusion of a Chemically Reactive Species in a Laminar Boundary Layer Flow", Phys. of Fluids. Vol. 1, no. 1, (1958), pp. 48-54.

9. Poonia, Hemant, and R. C. Chaudhary. "Mass transfer with chemical reaction effects on MHD free convective flow past an accelerated vertical plate embedded in a porous medium." Int. J. of Applied Mathematics \& Statistical Sciences 5 (2016).

10. Alexander Apelblat, "Mass Transfer with a Chemical Reaction of the First Order", Chem. Eng. J. vol. 19,(1980),pp. 19-37

11. U. N. Das, R. K. Deka, V. M. Soundalgekar, Effects of mass transfer on flow past an impulsively started infinite vertical plate with constant heat flux and chemical reaction, Forschung in Ingenieurwsen. 60 (1994) 284-287.

12. Job, VICTOR M., and S. Rao Gunakala."Unsteady MHD Free Convection Couette Flow between Two Vertical Permeable Plates in the Presence of Thermal Radiation Using Galerkin's Finite Element Method." International Journal of Mechanical Engineering 2.5 (2013): 99-110.

13. Dulal Pal andBabulalTalukdar, "Perturbation Analysis of Unsteady Magneto Hydrodynamic Convective Heat and Mass Transfer in a Boundary Layer Slip Flow Past a Vertical Permeable Plate with Thermal Radiation and Chemical Reaction”, Communications in Nonlinear Science and Numerical Simulation. vol. 15, no. 7,(2010), pp. 1813-1810.

14. S. P. Anjali Devi and J. Wilfred Samuel Raj, "Thermo Diffusion Effects on Unsteady Hydro Magnetic Free Convection Flow with Heat and Mass Transfer Past a Moving Vertical Plate with Time Dependent Suction and Heat Source in a Slip Flow Regime”, Int. J. of Appl. Math and Mech. vol. 7, no. 21, (2011), pp. 20-51

15. S. Masthanrao, K.S. Balamurugan, S.V.K. Varma and V.C.C. Raju, "Chemical Reaction and Hall Effects on MHD Convective Flow along an Infinite Vertical Porous Plate with Variable Suction and Heat Absorption”, Applications and Applied Mathematics: An International Journal (AAM), vol. 8, no. 1, (2013),pp. 268-288. 